\title{
A NOTE ON HOMOTOPY INVARIANCE OF TANGENT BUNDLES
}

\author{
HIROYASU ISHIMOTO
}

Although tangent bundles of manifolds are not always homotopically invariant, but in some categories of the manifolds they can be homotopically invariant.

In this note, I show that tangent bundles of $\pi$-manifolds and almost parallelizable manifolds depend only on their homotopy types.

We denote by $M^{n}$ a $n$-dimensional connected closed differentiable manifold, by $\tau M^{n}$ the tangent bundle, and by $\approx$ the bundle equivalence.

1. Let $M^{n}$ be a $\pi$-manifold. For a given immersion of $M^{n}$ into $(n+1)$ -dimensional Euclidean space or a triviality of the stable tangent bundle $\tau M^{n} \oplus \varepsilon$, we can define a map of $M^{n}$ into the $n$-dimensional sphere $S^{n}$, so called the "normal map" or the "Gauss map". This map is covered by a bundle map of $\tau M^{n}$ into $\tau S^{n}$, and its degree is decisively related to the Euler characteristic $\chi\left(M^{n}\right)$ and the semi-Euler characteristic of $M^{n}$. (Milnor [3], Bredon-Kosinski [1])

Theorem 1. Let $M_{1}^{n}$ and $M_{2}^{n}$ be $\pi$-manifolds of dimension $n$, and let $f$ : $M_{1}^{n} \rightarrow M_{2}^{n}$ be an arbitrary homotopy equivalence. Then, we have $\tau M_{1}^{n} \approx f^{*}\left(\tau M_{2}^{n}\right)$.

Proof. Let $F_{i}$ be a framing of the stable tangent bundle $\tau M_{i}^{n} \oplus \varepsilon$ and let $\nu_{F_{i}}$ be the Gauss map for $i=1,2$. Firstly we show that we can choose the framings $F_{1}$ and $F_{2}$ so that deg. $\nu_{F_{1}}=\operatorname{deg} \cdot \nu_{F_{2}}$.

If $n$ is even the assertion is clear, since deg. $\nu_{F_{1}}=\frac{1}{2} \chi\left(M_{1}^{n}\right), \operatorname{deg} \cdot \nu_{F_{2}}=$ $\frac{1}{2} \chi\left(M_{2}^{n}\right)$ independently of the choice of the framings and $M_{1}^{n}, M_{2}^{n}$ are of the same homotopy type.

For $n=1,3,7$, since $M_{1}^{n}$ and $M_{2}^{n}$ are parallelizable, the theorem is trivial. Let $n=2 r+1, n \neq 1,3,7$. By Theorem 3 of [1], deg. $\nu_{F_{i}} \equiv \sum_{k=0}^{r} \operatorname{rank}$

Received January 16, 1967. 
$H_{k}\left(M_{i}^{n} ; Z_{2}\right)$ mod. 2 for $i=1,2$, independently of the choice of the framings. Since $M_{1}^{n}$ and $M_{2}^{n}$ are of the same homotopy type, we know that $\operatorname{deg} \cdot \nu_{F_{1}} \equiv$ $\operatorname{deg} . \nu_{F_{2}} \bmod .2$ always.

Now, if we construct a new framing $F_{1}^{\prime}$ of $\tau M_{1}^{n} \oplus \varepsilon$ by a continuous map $g: M_{1}^{n} \rightarrow \mathrm{SO}(n+1)$, so that $F_{1}(x)=g(x) F_{1}{ }^{\prime}(x)$, there is a following relation between deg. $\nu_{F_{1}^{\prime}}$ and deg. $\nu_{F_{1}} \cdot((2 \cdot 2)$ of [1]).

deg. $\nu_{F_{1}^{\prime}}=\operatorname{deg} .(\pi \circ g)+$ deg. $\nu_{F_{1}}$, where $\pi$ denotes the canonical projection of $\mathrm{SO}(n+1)$ onto $S^{n}$. Thus we have,

deg. $\nu_{F_{1}^{\prime}}-\operatorname{deg} \cdot \nu_{F_{2}}=$ deg. $(\pi \circ g)+\left(\right.$ deg. $\nu_{F_{1}}-$ deg. $\left.\nu_{F_{2}}\right)$. We note that $\operatorname{deg} \cdot \nu_{F_{1}}-\operatorname{deg} \cdot \nu_{F_{2}}$ is even. If $n$ is odd, since there exists a continuous map $g$ such that deg. $(\pi \circ g)$ can be any even integer, we can choose $F_{1}^{\prime}$ so that $\operatorname{deg} \cdot \nu_{F_{1}^{\prime}}=\operatorname{deg} \cdot \nu_{F_{2}}$.

Thus we can choose the framings $F_{1}$ and $F_{2}$ so that deg. $\nu_{F_{1}}=$ $\operatorname{deg} \cdot \nu_{F_{2}}$. We may assume that deg. $f=1$. Then, in the following diagram, deg. $\nu_{F_{2}} \circ f=$ deg. $f \times \operatorname{deg} \cdot \nu_{F_{2}}=\operatorname{deg} \cdot \nu_{F_{2}}=$ deg. $\nu_{F_{1}}$. So that by Hopf's $M_{1}^{n} \nu_{F_{1}} \begin{aligned} & \text { Classification Theorem, } \nu_{F_{2}} \circ f \text { is homotopic to } \nu_{F_{1}}, \\ & \text { that is, the diagram is homotopy commutative. There- } \\ & \text { fore we have } \tau M_{1}^{n} \approx \nu_{F_{1}}^{*}\left(\tau S^{n}\right) \approx\left(\nu_{F_{2}} \circ f\right)^{*}\left(\tau S^{n}\right) \approx f^{*}\left(\nu_{F_{2}}^{*}\left(\tau S^{n}\right)\right) \\ & \approx f^{*}\left(\tau M_{2}^{n}\right) .\end{aligned}$

This completes the proof of the theorem.

In the proof of this theorem, if $\chi\left(M_{1}^{n}\right)=\chi\left(M_{2}^{n}\right)$ or if the semi-Euler characteristic of $M_{1}^{n}$ is equal to that of $M_{2}^{n}$ in mod.2, according as $n$ is even or odd, then the map $f$ need not be a homotopy equivalence but the condition deg. $f=1$. So, we have

Theorem $1^{\prime}$. Let $M_{1}^{n}$ and $M_{2}^{n}$ be $\pi$-manifolds of dimension $n$. If $n$ is even and $\chi\left(M_{1}^{n}\right)=\chi\left(M_{2}^{n}\right)$, or if $n$ is odd and the semi-Euler characteristic of $M_{1}^{n}$ is equal to that of $M_{2}^{n}$ in mod. 2 , then for any continuous map $f: M_{1}^{n} \rightarrow M_{2}^{n}$ of degree 1 , $\tau M_{1}^{n} \approx f^{*}\left(\tau M_{2}^{n}\right)$.

COROLlaRy 1. In the category of $\pi$-manifolds, whether a $\pi$-manifold of even dimension has an almost complex structure or not depends only on its homotopy type.

Proof. This is clear.

Corollary 2. Let $M_{1}^{n}$ and $M_{2}^{\prime \prime}$ be $\pi$-manifolds of dimension $n$, and let $f$ : $M_{1}^{n} \rightarrow M_{2}^{n}$ be a homotopy equivalence. Then, for any twisted spheres $T_{1}$ and $T_{2}$ of 
$\Gamma^{n}$, there exists a homotopy equivalence $g: M_{1}^{n} \# T_{1} \rightarrow M_{2}^{n} \sharp T_{2}$ such that $\tau\left(M_{1}^{n} \# T_{1}\right) \approx$ $g^{*} \tau\left(M_{2}^{n}+T_{2}\right)$.

Proof. Since $M_{i}^{n} \# T_{i} i=1,2$ are $\pi$-manifolds and are homeomorphic to $M_{i}^{n}$, this follows from Theorem 1 .

2. Let us consider such a manifold $M^{n}$ that $M^{n}-$ (a point) is an open $\pi$-manifold. If we call such a manifold to be almost $\pi$, a manifold $M^{n}$ is almost $\pi$ if and only if it is almost parallelizable. Because, the tangent bundle of $M^{n}$ - (a point) is induced from that of $M^{n}$-(Interior of an imbedded $n$-disk), and a manifold with boundary is $\pi$ if and only if it is parallelizable. (Kervaire-Milnor [5]).

Theorem 2. Let $M_{1}^{n}$ and $M_{2}^{n}$ be almost parallelizable manifolds, and let $f$ : $M_{1}^{n} \rightarrow M_{2}^{n}$ be a homotopy equivalence. Then, we have that $\tau M_{1}^{n} \approx f^{*}\left(\tau M_{2}^{n}\right)$. In other words, tangent bundles of $(n-1)$-parallelizable manifolds are homotopically invariant.

Proof. Let $O_{i} \in H^{n}\left(M_{i}^{n} ; \pi_{n-1}\left(S O_{n+1}\right)\right) \quad i=1,2$. be the obstruction class for extending the triviality of $\tau M_{i}^{n} \oplus \varepsilon$ on the $(n-1)$-skeleton over the whole. If $n \equiv 1,2,3,5,6,7$ mod. 8, by the analogous argument of Kervaire and Milnor [4], [5], we know that $M_{1}^{n}$ and $M_{2}^{n}$ are $\pi$-manifolds. So, the theorem is valid by Theorem 1 . If $n=4 k$, since the $k$-th Pontrjagin classes $P_{k}\left(M_{i}^{n}\right)=m O_{i}$ ( $m$ : an integer) $i=1,2$ and the indexes of $M_{1}^{n}$ and $M_{2}^{n}$ are equal (we may assume that deg. $f=1$.), we know that $O_{1}=f^{*} O_{2}$. So that, the obstruction class $f^{*} O_{2}-O_{1}$ for extending the isomorphism of $\tau M_{1}^{n} \oplus \varepsilon$ onto $f^{*}\left(\tau M_{2}^{n}\right) \oplus \varepsilon$ on the $(n-1)$-skeleton over the whole vanish. Thus, $f^{*}\left(\tau M_{2}^{n}\right)$ is stably equivalent to $\tau M_{1}^{n}$. But, in this case, we can show that $f^{*}\left(\tau M_{2}^{n}\right)$ is equivalent to $\tau M_{1}^{n}$; If we denote by $\alpha_{i} \in H^{n}\left(M_{i}^{n} ; \pi_{n-1}\left(S O_{n}\right)\right)$ $i=1,2$ the obstruction classes for extending the triviality of $\tau M_{i}^{n}$ on the $(n-1)$-skeleton over the whole, then the obstruction class for extending the isomorphism of $\tau M_{1}^{n}$ onto $f^{*}\left(\tau M_{2}^{n}\right)$ on the $(n-1)$-skeleton is given by $f^{*} \alpha_{2}-\alpha_{1}$. we can show that $f^{*} \alpha_{2}-\alpha_{1}=0$. The proof is included in that of K. Shiraiwa [7].

Finally, note that if $M^{n}$ is a $(n-1)$-parallelizable manifold, then $M^{n}$ is almost parallelizable. Because, choose a point of $M^{n}$ and tie to an interior point of every $n$-simplex with an imbedded arc. Then, there exists a $n$-cell which contains the tree. 
This completes the proof.

CoROllaRy 3. For $(n-1)$-connected $2 n$-manifolds, $n \equiv 3,5,6,7$ mod. 8 , their tangent bundles are homotopically invariant. For $(n-1)$-connected $(2 n+1)$-manifolds, $n \equiv 5,6 \bmod .8$, their tangent bundles are homotopically invariant.

Proof. In this case, these manifolds are almost parallelizable or stably parallelizable.

COROLlary 4. The matters corresponding to the corollaries 1,2 are also valid for almost parallelizable manifolds.

\section{REFERENCES}

[1] G.E. Bredon and A. Kosinski, Vector fields on $\pi$-manifolds, Ann. of Math., 84 (1966), 85-90.

[2] Wu-chung Hsiang, A note on free differentiable actions of $S^{1}$ and $S^{3}$ on homotopy spheres, Ann. of Math., 83 (1966), 266-272.

[3] J. Milnor, On the immersion of n-manifolds in $(n+1)$-space, Comment. Math. Helv., 30 (1955), 275-284.

[4] — and M. Kervaire, Bernoulli numbers, homotopy groups and a theorem of Roholin, Proc. Int. Congress of Math., Edinburgh, 1958.

[5] M. Kervaire and J. Milnor, Groups of homotopy spheres, I, Ann. of Math., 77 (1963), 504-537.

[6] K. Shiraiwa, A note on tangent bundles, Nagoya Math. Jour., to appear.

[7] - A note on tangential equivalences., to appear.

[8] N.E. Steenrod, The topology of fibre bundles, Princeton, 1951.

Kanazazea University. 